\title{
Practice Patterns of Inferior Vena Cava Filter Placement and Factors That Predict Retrieval Rates: A Single-Center Institution and Review of the Literature
}

\author{
Ming Y. Lim ${ }^{\mathrm{a}, \mathrm{c}}$, Ricardo Yamada ${ }^{\mathrm{b}}$, Marcelo Guimaraes ${ }^{\mathrm{b}}$, Charles S. Greenberg ${ }^{\mathrm{a}}$
}

\begin{abstract}
Background: There is a wide variability in practice patterns on the use of inferior vena cava filters (IVCFs) among institutions, which is likely due to contrasting indication guidelines published by different professional societies. The aim of the present study is to report our healthcare system use of IVCF to: 1) determine practice patterns, 2) determine factors that may predict IVCF retrieval and 3) identify areas for improvement.
\end{abstract}

Methods: A retrospective review of 180 consecutive IVCF placement performed between July 2014 and December 2015 was conducted.

Results: One hundred nine (60.6\%) IVCFs were placed for absolute indications, 27 (15.0\%) for relative indications, 26 (14.4\%) prophylactically and $18(10.0 \%)$ for unknown indications. Average age was 59.3 years. Ninety-five had active cancer. Surgical and medical services requested filter placement in $112(62.2 \%)$ and $68(37.8 \%)$ patients, respectively. Thirteen $(7.2 \%)$ patients had a hematology consult prior to IVCF placement. Documentation of the presence of an IVCF was present in 118/127 (92.9\%) discharge summaries, and outlined instructions for filter retrieval post-discharge were present in 20/124 (16.1\%) cases. Only $33(25.0 \%)$ IVCF were retrieved at a median interval of 162 days (range: 4 - 1,053 days). None of the factors of interest was found to be significantly associated with IVCF retrieval.

Conclusion: A root cause analysis identified that the lack of a structured system for IVCF tracking resulted in poor IVCF retrieval rates. This study resulted in the development of a hospital-initiated multidisciplinary team to address these issues.

Keywords: Thromboembolism; Clinical practice guidelines; High

Manuscript submitted July 25, 2018, accepted August 20, 2018

aDepartment of Medicine, Division of Hematology/Oncology, Medical University of South Carolina, Charleston, SC, USA

bivision of Vascular and Interventional Radiology, Medical University of South Carolina, Charleston, SC, USA

${ }^{\mathrm{c} C}$ Corresponding Author: Ming Y. Lim, Medical University of South Carolina, 39 Sabin Street, MSC 635, Charleston, SC 29425, USA.

Email:mylim@cantab.net

doi: https://doi.org/10.14740/jocmr3544w value care; Quality improvement

\section{Introduction}

In large academic medical centers, there are multiple disciplines and clinical scenarios that may require placement of inferior vena cava filters (IVCFs). Both the Society of Interventional Radiology (SIR) and the American College of Chest Physicians (ACCP) have released guidelines for IVCF use, with the ACCP guidelines being more stringent due to the lack of survival benefit supported by level I data (Tables 1 and 2) [1,2]. Several publications have reported high rates of retrievable IVCF complications [3-5]. In response to these risks and low retrieval rates, the US Food and Drug Administration issued an updated safety alert in 2014 recommending that implanting physicians and clinicians responsible for the ongoing care of patients with retrievable IVCF consider removing the filter as soon as protection from pulmonary embolism is no longer needed (https://wayback.archive-it. org/7993/20161022044053/http://www.fda.gov/MedicalDevices/Safety/AlertsandNotices/ucm396377.htm).

As a first step, the purpose of this study was to report our healthcare system use of IVCF to: 1) determine practice patterns, 2) determine factors that may predict IVCF retrieval and 3) identify areas for improvement.

\section{Patients and Methods}

Institutional review board approval was obtained to perform a retrospective review of hospital medical records of consecutive patients who had an IVCF placed by Vascular Interventional Radiology (VIR) between July 2014 and December 2015 at the Medical University of South Carolina.

\section{Data collection}

Patients who had an IVCF placed by VIR were identified by the inventory tracking data kept by the radiology business manager. Electronic medical records were reviewed in June 
Table 1. Society of Interventional Radiology Guidelines for Indications for Inferior Vena Cava Filters
Absolute indications
Recurrent VTE (acute or chronic) despite adequate anticoagulation
Contraindication to anticoagulation
Complication of anticoagulation
Inability to achieve/maintain therapeutic anticoagulation
Relative indications
Iliocaval DVT
Large, free-floating proximal DVT
Difficulty establishing therapeutic anticoagulation
Massive PE treated with thrombolysis/thrombectomy
Chronic PE treated with thromboendarterectomy
Thrombolysis for iliocaval DVT
VTE with limited cardiopulmonary reserve
Recurrent PE with filter in place
Poor compliance with anticoagulant medications
High risk of complication of anticoagulation (e.g. ataxia, frequent falls)
Prophylactic indications
Trauma patient with high risk of VTE
Surgical procedure in patient at high risk of VTE
Medical condition with high risk of VTE

DVT: deep venous thrombosis; PE: pulmonary embolism; VTE, venous thromboembolism.

2016 and followed until August 2017. Data extraction included the following: patient demographics, referring services, presence of cancer, hematology consult prior to IVCF placement, indications for IVCF placement, types of IVCF placed, complications at insertion and for the duration the IVCF remained in situ, documentation of IVCF placement and instructions for retrieval on discharge summary (if applicable), discharge location, outpatient follow-up with hematology and time to IVCF retrieval and complications (if any). The type of IVCF placed (permanent versus retrievable) was at the discretion of VIR. Insertion indications were analyzed based on the established guidelines by SIR and ACCP $[1,2]$.

\section{Statistical analysis}

Descriptive statistics were used on patients and IVCF demographics. Mean and range were used for continuous variables, whereas count and frequency (\%) were presented for categorical variables. Patients who died or were discharge to hospice and those with permanent IVCF were not included in the anal- ysis for retrieval rates. The odds ratio was used to compare the relative odds of IVCF retrieval given exposure to the variables of interest. The variables of interest were determined a priori to be hematology consultation pre-IVCF placement, type of services requesting IVCF placement (medical versus surgical), documented instruction for IVCF retrieval on the discharge summary and discharge location (home versus non-home). Statistical significance was determined using an $\alpha$ level of $\mathrm{P}$ $<0.05$.

\section{Results}

\section{Patient demographics}

During the study period, 180 consecutive patients underwent IVCF placement by VIR at our institution with a $100 \%$ technical success rate. The average age at time of placement was 59.3 years (range: 19 - 85 years), and 83 (46.1\%) were male. The majority of IVCF placements were performed inpatient

Table 2. American College of Chest Physician Guidelines for Indications for Inferior Vena Cava Filters

For the initial treatment of patients with acute proximal DVT or PE, if anticoagulant therapy is not possible because of the risk of bleeding, placement of an IVCF is recommended (grade 1C)

For patients with chronic thromboembolic pulmonary hypertension (CTPH) undergoing pulmonary thromboendarterectomy, placement of a permanent vena caval filter before or at the time of the procedure is suggested (grade 2C) 
Table 3. Referring Services Requesting IVCF Placement

\begin{tabular}{|c|c|}
\hline \multicolumn{2}{|l|}{ Referring services } \\
\hline Medical services & n \\
\hline General medicine & 23 \\
\hline Neurology/neurology intensive care unit/stroke & 16 \\
\hline Oncology & 10 \\
\hline Malignant hematology & 9 \\
\hline Medical intensive care unit & 5 \\
\hline Gastroenterology & 3 \\
\hline Cardiology & 1 \\
\hline Primary care & 1 \\
\hline Total & $68(37.8 \%)$ \\
\hline Surgical services & n \\
\hline Gynecology oncology & 37 \\
\hline Neurosurgery & 18 \\
\hline Trauma surgery & 10 \\
\hline General/acute surgery & 8 \\
\hline Gastroenterology surgery & 7 \\
\hline Orthopedics & 6 \\
\hline Surgical oncology & 6 \\
\hline Renal transplant & 4 \\
\hline Urology & 4 \\
\hline Surgical intensive care unit & 4 \\
\hline Ear, nose and throat & 2 \\
\hline Vascular surgery & 2 \\
\hline Cardiothoracic surgery & 1 \\
\hline Heart transplant & 1 \\
\hline Obstetrics & 1 \\
\hline Thoracic surgery & 1 \\
\hline Total & $112(62.2 \%)$ \\
\hline
\end{tabular}

( $n=160,88.9 \%)$. About half of the patients had active cancer $(n=95,52.83 \%)$. Surgical and medical services requested filter placement in $112(62.2 \%)$ and $68(37.8 \%)$ patients, respectively. The referring services requesting filter placement are shown in Table 3 . Only $13(7.2 \%)$ patients had a hematology consult prior to IVCF placement.

\section{Filter insertion indication and characteristics}

Of the 180 IVCFs inserted successfully, 26 were permanent and 154 were retrievable. Overall, $109(60.6 \%)$ IVCFs were placed for absolute indications as per both ACCP and SIR guidelines, $27(15.0 \%)$ for relative indications as per SIR guidelines, 26 (14.4\%) prophylactically and $18(10.0 \%)$ with no clear indications. Demographics, insertion indication and discharge location for permanent and retrievable IVCF are summarized in Tables 4 and 5, respectively.
Table 4. Detailed Analysis of Permanent IVCF Placement

\begin{tabular}{lll}
\hline Permanent IVCF placement & $\mathbf{n}=\mathbf{2 6}$ & $\mathbf{\%}$ \\
\hline Mean age (years) & 71.1 & \\
Range (years) & $54-85$ & 73.1 \\
\hline Presence of cancer & 19 & \\
Indication for placement & & \\
Contraindication to AC & 19 \\
$\quad$ Bleeding & 13 \\
$\quad$ Intracranial hemorrhage & 2 \\
$\quad$ Surgery & 3 \\
$\quad$ Thrombocytopenia & 1 \\
Recurrent VTE despite adequate AC & 3 \\
High risk of complication of AC & 1 \\
\hline Prophylaxis - history of VTE & 2 \\
None & 1 \\
\hline Died inpatient & 3 \\
Discharge to home hospice & 2 \\
Of the remaining 21 patients & \\
Restarted AC prior to discharge & 8 \\
\hline
\end{tabular}

IVCF: inferior vena cava filter; AC: anticoagulation; VTE: venous thromboembolism.

\section{Filter documentation and follow-up management}

Of the 154 retrievable IVCFs, 22 patients died during their hospitalization or were discharged to home hospice. Of the remaining 132 cases, five cases were performed as an outpatient. The documentation of the presence of an IVCF was present in $118 / 127(92.9 \%)$ discharge summaries. Outlined instructions for filter retrieval post-discharge were present in 20/124 (16.1\%) cases; three IVCFs were removed during the same hospitalization.

While many of the retrievable filters were placed because of an absolute contraindication $(n=71)$ or relative indication for high-risk of complication to anticoagulation $(n=13), 44$ $(55.7 \%)$ patients were initiated and discharged on a regimen of anticoagulant therapy in the same hospitalization.

\section{Filter retrieval}

Only $33(25.0 \%)$ IVCFs were retrieved at a median interval of 162 days (range: 4 - 1,053 days) at the time this study was completed (August 2017). Of the 23 patients that had IVCF placed prophylactically (excluding patients that died during their hospitalization or were discharged to home hospice), only nine $(39.1 \%)$ IVCFs were retrieved.

\section{Complications}

One patient experienced immediate complication post-filter 
Table 5. Detailed Analysis of Retrieval IVCF Placement

\begin{tabular}{|c|c|c|}
\hline Retrievable IVCF placement & $n=154$ & $\%$ \\
\hline Mean age (years) & 57.3 & \\
\hline Range (years) & $19-84$ & \\
\hline Presence of cancer & 76 & 49.4 \\
\hline \multicolumn{3}{|l|}{ Indication for placement } \\
\hline Contraindication to AC & 84 & \\
\hline Bleeding & 39 & \\
\hline Intracranial hemorrhage & 16 & \\
\hline Surgery & 24 & \\
\hline Thrombocytopenia & 5 & \\
\hline Recurrent VTE despite adequate AC & 2 & \\
\hline Inability to maintain $\mathrm{AC}$ & 1 & \\
\hline High risk of complication of $\mathrm{AC}$ & 15 & \\
\hline VTE with limited CV reserve & 2 & \\
\hline Thrombectomy for DVT & 3 & \\
\hline Thrombolysis for PE & 4 & \\
\hline Iliac caval or free floating DVT & 2 & \\
\hline Prophylaxis - no history of VTE & 8 & \\
\hline Prophylaxis - history of VTE & 16 & \\
\hline None & 17 & \\
\hline \multicolumn{3}{|l|}{ Discharge locations } \\
\hline Died inpatient & 11 & \\
\hline Home hospice & 11 & \\
\hline Acute care hospital/LTAC & 14 & \\
\hline Rehab unit/SNF/nursing home & 37 & \\
\hline Home & 80 & \\
\hline Acute psychiatric unit & 1 & \\
\hline
\end{tabular}

IVCF: inferior vena cava filter; AC: anticoagulation; VTE: venous thromboembolism; CV: cardiovascular; DVT: deep vein thrombosis; PE: pulmonary embolism; LTAC: long-term acute care; SNF: skilled nursing facility.

insertion. The patient became hemodynamically unstable at the end of the procedure, requiring intensive care monitoring. Following placement of an IVCF, three patients developed subsequent thrombotic events: one acute right posterior tibial and peroneal deep vein thrombosis (DVT) after 3 days, one subsegmental pulmonary embolism (PE) after 4 days and one IVC thrombus after 8 days. No complications of filter retrieval were documented.

\section{Predictors for filter retrieval}

None of the factors of interest was found to be significantly associated with IVCF retrieval (Table 6).

\section{Discussion}

This study shows that about $60 \%$ of IVCF placements were compliant with both ACCP and SIR guidelines, whereas about $30 \%$ were placed for relative indications as per SIR guidelines alone. This is somewhat reassuring that the majority of IVCF placement was placed according to society guidelines. However, only $25 \%$ of retrievable IVCFs were removed. This is consistent with published studies with retrieval rates ranging from $12 \%$ to $45 \%$ [3]. Reasons for poor retrieval rates have been attributed to transition of care resulting in oversight, poor discharge documentation, poor provider-to-provider communication and poor follow-up management [6-8]. One Australian retrospective study found that the lack of hematology outpatient clinic follow-up was significantly associated with loss to follow-up [6]. This was further confirmed by Tao et al who estimated that in patients with thrombosis, a clinic follow-up were 6.7 times as likely to have their filter removed, as compared to those who did not have follow-up $(\mathrm{P}<0.01)$ [9].

As such, there have been lots of studies that focused on improving overall retrieval rates with the use of IVCF followup clinics and personnel dedicated to ensuring follow-up care [8, 10-13]. Such efforts have had varying success. Using an IVCF retrieval protocol with a multidisciplinary team approach, Inagaki et al improved the retrieval rate of IVCF from $11 \%$ to $54 \%$ [14]. In almost all of the studies looking at methods to improve retrieval rates, there are very limited studies looking at the importance of patient education in this setting. One study implemented a multidisciplinary quality improvement program that included initial verbal counseling and printed educational materials, correspondence after discharge and a hematology consultation. This approach was able to improve their IVCF retrieval rates from $23 \%$ to $45 \%$ [15].

Despite all these efforts, the retrieval rate remains low. One possible reason could be transition of care. Tao et al reported that fewer than expected number of removals was seen in those referred to long-term care compared with those discharged home [9]. In our study, we failed to find a difference in IVCF retrieval rates in patients discharged home as compared to non-home (i.e. acute rehab, acute care hospital) but this is likely due to the low sample size. There is often a communication gap during institution transfer resulting in subop-

Table 6. Odds Ratio for Predictors of IVCF Retrieval

\begin{tabular}{llll}
\hline Variable & OR & 95\% CI & P value \\
\hline Prior hematology consultation & 3.96 & $0.92-1.69$ & 0.0635 \\
Surgical services (compared with medical services) & 2.43 & $0.91-6.50$ & 0.0758 \\
Outlined instructions on discharge summary & 2.41 & $0.85-6.85$ & 0.0991 \\
Discharge to home (compared to non-home locations) & 2.12 & $0.81-5.51$ & 0.1222 \\
\hline
\end{tabular}


timal follow-up and subsequent filter retrieval. Alternatively, patients may be discharged to relatives' home, thus making it difficult to locate these patients. This makes sense as ensuring that appropriate follow-up is dependent on being able to locate the patient.

In our study, the documentation of IVCF placement was present in $92.2 \%$ of discharges summaries, but only $16.1 \%$ had outlined instructions for filter retrieval. It is possible that instructions given at time of discharge may prompt long-term care providers and/or patients to seek timely follow-up, whereas attempting to locate these patients post-discharge when their contact information is no longer up-to-date results in lost to follow-up. However, we failed to find a difference in IVCF retrieval rates when specific discharge instructions for IVCF retrieval were provided.

Even with best efforts in follow-up, studies have shown that retrieval rates remain low [16]. As part of a quality improvement project, the Vascular Surgery fellowship program at Walter Reed National Military Medical Center implemented a registry to track IVCFs placed to improve retrieval rates [16]. They were able to improve their follow-up rate to $94 \%$. Even with a $>90 \%$ follow-up rate, around a third of IVCFs were not retrieved during the 38-month study period. Given these findings, it may be more beneficial to focus on appropriate indications for IVCF placement.

In our study, about a quarter of retrieval IVCFs were placed prophylactically or for no clear indication. Currently, the use of prophylactic IVCF is predominantly in the trauma, orthopedic and bariatric surgical populations; however, the benefits remain controversial. Using a propensity-matched cohort study, the Michigan Bariatric Surgery Collaborative published data from their statewide clinical registry and found that IVCF patients had significantly higher rates of venous thromboembolism ( $1.9 \%$ versus $0.74 \%$; OR: 2.7 ; $95 \%$ CI: 1.1 - 6.3 ; $\mathrm{P}=0.027)$ and deep vein thrombosis $(1.2 \%$ versus $0.37 \%$, OR: 3.3; 95\% CI: $1.1-10.1 ; \mathrm{P}=0.039)$ than matched control patients [17]. Prophylactic IVCF placement was not associated with a reduction in the rate of $\mathrm{PE}(0.84 \%$ versus $0.46 \%$; OR: 2.0; 95\% CI: $0.6-6.5 ; \mathrm{P}=0.232$ ) [17].

Hemmila et al analyzed trauma quality collaborative data from 2010 to 2014 and found that prophylactic IVCF placement had no effect on reducing trauma patient mortality, but was associated with an increase in DVT events (OR: 1.83, $95 \%$ CI: $1.15-2.93, \mathrm{P}=0.01$ ) [18]. Sarosiek et al performed a retrospective cohort study of patients who underwent IVCF placement at a level 1 trauma center and found no significant difference in survival in trauma patients with or without placement of an IVCF, whether in the presence or absence of venous thrombosis [19]. As such, they call for re-examination of IVCF use in this patient population [19].

Similarly, in patients with traumatic brain injury, the authors of the Parkland Protocol, an algorithm for venous thromboembolism (VTE) prophylaxis after traumatic brain injury, noted that the rate of symptomatic PE at their institution remained similar, despite a change in their IVCF placement policy from very liberal to very restrictive [20]. As a result, they dropped their previous recommendations for prophylactic IVCF use in high-risk patients from their most recent iteration of the Parkland Protocol [20]. A recent meta-analysis of prophylactic IVCF efficacy found that no class I studies exist to support prophylactic IVCF use, and such use should be discouraged [21]. These studies support the recommendation by the American College of Chest Physicians against IVCF placement in high-risk patients without VTE [22].

In contrast, the SIR recommends placement of IVCF in high-risk trauma, surgical and medical patients without VTE who have contraindications to standard DVT pharmacological prophylaxis [1]. Supporting this recommendation is a metaanalysis of eight controlled single institution studies in North America that found an association between IVCF placement and reduction of PE (relative risk: 0.20 ; 95\% CI: $0.06-0.70$ ) and fatal PE (relative risk: $0.09 ; 95 \%$ CI: $0.01-0.81$ ) in the trauma patient population in whom filters were placed [23]. There was no significant difference in incidence of DVT (relative risk: $1.76 ; 95 \%$ CI: $0.50-6.19$ ) or mortality (relative risk: $0.70 ; 95 \%$ CI: $0.40-1.23$ ]) [23]. Given the variability of existing data, it is not surprising that hospital practice patterns vary widely from $0.6 \%$ to $9.6 \%$ in regard to prophylactic IVCF use in this population [18].

A major concern in our study is the low retrieval rate for IVCFs placed for prophylactic indications with only $39.0 \%$ retrieved. One would assume that the percentage of retrieval attempts should be higher because nearly all should be retrieval candidates. This low retrieval rate strongly highlights the need for a proper vetting process for whether IVCF placement is needed. As there is currently insufficient evidence to support the use of IVCF for prophylactic indications and the often reported low retrieval rates, it may be best to avoid placement of such IVCFs for these indications.

This study shows that only $7 \%$ of patients had a hematology consult prior to IVCF placement. However, this could be explained by the possibility that patients that had a hematology consult ended up not having an IVCF placed. We were not able to track this group of people. A hematology consultation was associated with higher rates of IVCF documentation and was a significant predictor of retrieval attempts [24]. Although we did not find a difference in IVCF retrieval rates with hematology consultation in our study, we strongly emphasize the importance of the hematology consult service. Hematologists working collaboratively with VIR physicians tend to have heightened awareness of filter indication and complications, which translates into enhanced diligence in advocating for appropriate filter placement and removal.

Almost half of our patients resumed anticoagulation therapy before being discharged. As such, these patients qualified to have their filter removed during the hospitalization, which would help reduce the barrier of lost to follow-up. However, this creates a separate issue in terms of hospital reimbursement. IVCF retrieval on the same hospital admission would not increase reimbursement, whereas outpatient retrieval is reimbursed separately. Whether this contributes to the low rate of IVCF retrieval during the same hospitalization is unknown.

This study had a few limitations. This study was a retrospective chart review and was subjected to the accuracy of documentation by the medical and surgical teams. As we are a tertiary referral center, most of our patients are referred back to their local primary care providers for follow-up. Some of our patients could have had their filters removed locally; thus, 
these retrievals were not documented in our study, resulting in a low retrieval rate. In addition, this study only looks at IVCF placement by VIR due to availability of a departmental inventory tracking list. Other clinical services such as Vascular Surgery, Trauma Surgery and Interventional Cardiology are also involved in IVCF placement and were not reviewed in this study. The number of IVCF placed by these clinical services contributes to about $10-15 \%$ of total IVCF placed at our institution. Another limitation of this study is the small sample size that likely resulted in non-statistical significant predictors of IVCF retrievals.

\section{Conclusions}

Our study confirms that our institution is adhering to either ACCP and/or SIR guidelines for indication for IVCF placement but could improve on the prophylactic use of IVCF. More importantly, IVCF retrieval rates remain low at our institution. As a result of this study, we performed a root cause analysis and identified that the lack of a structured system for IVCF tracking resulted in poor IVCF retrieval rates. This was brought to the attention of the Chief Quality Officer at our institution. With institutional support, a hospital-initiated multidisciplinary team comprising of Hematologist, Interventional Cardiologist, Vascular Interventional Radiologist, Intensivist and Vascular Surgeon has been created to develop a protocol to optimize filter placement and retrieval rates. An action plan was implemented, including creation of a shared database including pertinent patient information, standardization of follow-up protocol (three attempts to contact the patient by phone call, followed by mailed letter, all documented in electronic medical records), creation of educational brochure to be given to the patient or family member when obtaining informed consent, and monthly analysis of the data. The outcome of this action plan will be analyzed for future studies.

\section{Conflict of Interest}

The authors declare that they have no conflict of interest.

\section{Financial Support}

None.

\section{References}

1. Kaufman JA, Kinney TB, Streiff MB, Sing RF, Proctor $\mathrm{MC}$, Becker D, Cipolle M, et al. Guidelines for the use of retrievable and convertible vena cava filters: report from the Society of Interventional Radiology multidisciplinary consensus conference. J Vasc Interv Radiol. 2006;17(3):449-459.

2. Kearon C, Akl EA, Ornelas J, Blaivas A, Jimenez D, Bounameaux H, Huisman M, et al. Antithrombotic Therapy for VTE Disease: CHEST Guideline and Expert Panel Report. Chest. 2016;149(2):315-352.

3. Angel LF, Tapson V, Galgon RE, Restrepo MI, Kaufman J. Systematic review of the use of retrievable inferior vena cava filters. J Vasc Interv Radiol. 2011;22(11):15221530 e 1523.

4. Tam MD, Spain J, Lieber M, Geisinger M, Sands MJ, Wang W. Fracture and distant migration of the Bard Recovery filter: a retrospective review of 363 implantations for potentially life-threatening complications. J Vasc Interv Radiol. 2012;23(2):199-205 e191.

5. Andreoli JM, Lewandowski RJ, Vogelzang RL, Ryu RK. Comparison of complication rates associated with permanent and retrievable inferior vena cava filters: a review of the MAUDE database. J Vasc Interv Radiol. 2014;25(8):1181-1185.

6. Tan XL, Tam C, McKellar R, Nandurkar H, Bazargan A. Out of sight, out of mind: an audit of inferior vena cava filter insertion and clinical follow up in an Australian institution and literature review. Intern Med J. 2013;43(4):365-372.

7. Albrecht RM, Garwe T, Carter SM, Maurer AJ. Retrievable inferior vena cava filters in trauma patients: factors that influence removal rate and an argument for institutional protocols. Am J Surg. 2012;203(3):297-302; discussion 302.

8. Ko SH, Reynolds BR, Nicholas DH, Zenati M, Alarcon L, Dillavou ED, Chaer R, et al. Institutional protocol improves retrievable inferior vena cava filter recovery rate. Surgery. 2009;146(4):809-814; discussion 814-806.

9. Tao MJ, Montbriand JM, Eisenberg N, Sniderman KW, Roche-Nagle G. Temporary inferior vena cava filter indications, retrieval rates, and follow-up management at a multicenter tertiary care institution. J Vasc Surg. 2016;64(2):430-437.

10. Minocha J, Idakoji I, Riaz A, Karp J, Gupta R, Chrisman HB, Salem R, et al. Improving inferior vena cava filter retrieval rates: impact of a dedicated inferior vena cava filter clinic. J Vasc Interv Radiol. 2010;21(12):1847-1851.

11. O'Keeffe T, Thekkumel JJ, Friese S, Shafi S, Josephs SC. A policy of dedicated follow-up improves the rate of removal of retrievable inferior Vena Cava Filters in trauma patients. Am Surg. 2011;77(1):103-108.

12. Gasparis AP, Spentzouris G, Meisner RJ, Elitharp D, Labropoulos $\mathrm{N}$, Tassiopoulos A. Improving retrieval rates of temporary inferior vena cava filters. J Vasc Surg. 2011;54(6 Suppl):34S-38S e31.

13. Ryu RK, Parikh P, Gupta R, Eifler AC, Salem R, Omary RA, Lewandowski RJ. Optimizing IVC filter utilization: a prospective study of the impact of interventional radiologist consultation. J Am Coll Radiol. 2012;9(9):657-660.

14. Inagaki E, Farber A, Eslami MH, Siracuse JJ, Rybin DV, Sarosiek S, Sloan JM, et al. Improving the retrieval rate of inferior vena cava filters with a multidisciplinary team approach. J Vasc Surg Venous Lymphat Disord. 2016;4(3):276-282.

15. Winters JP, Morris CS, Holmes CE, Lewis P, Bhave AD, Najarian KE, Shields JT, et al. A multidisciplinary quality improvement program increases the inferior vena cava 
filter retrieval rate. Vasc Med. 2017;22(1):51-56.

16. Simon TE, Walker PF, Daab LJ, White JM, White PW. A quality improvement project to improve inferior vena cava filter retrieval. J Vasc Surg Venous Lymphat Disord. 2017;5(1):42-46.

17. Birkmeyer NJ, Finks JF, English WJ, Carlin AM, Hawasli AA, Genaw JA, Wood MH, et al. Risks and benefits of prophylactic inferior vena cava filters in patients undergoing bariatric surgery. J Hosp Med. 2013;8(4):173-177.

18. Hemmila MR, Osborne NH, Henke PK, Kepros JP, Patel SG, Cain-Nielsen AH, Birkmeyer NJ. Prophylactic inferior vena cava filter placement does not result in a survival benefit for trauma patients. Ann Surg. 2015;262(4):577585.

19. Sarosiek S, Rybin D, Weinberg J, Burke PA, Kasotakis G, Sloan JM. Association Between Inferior Vena Cava Filter Insertion in Trauma Patients and In-Hospital and Overall Mortality. JAMA Surg. 2017;152(1):75-81.

20. Pastorek RA, Cripps MW, Bernstein IH, Scott WW, Madden CJ, Rickert KL, Wolf SE, et al. The Parkland Protocol's modified Berne-Norwood criteria predict two tiers of risk for traumatic brain injury progression. J Neurotrauma. 2014;31(20):1737-1743.

21. Davies MG, Hart JP, El-Sayed HF. Efficacy of prophylactic inferior vena caval filters in prevention of pulmonary embolism in the absence of deep venous thrombosis. J Vasc Surg Venous Lymphat Disord. 2016;4(1):127-130 e121.

22. Gould MK, Garcia DA, Wren SM, Karanicolas PJ, Arcelus JI, Heit JA, Samama CM. Prevention of VTE in nonorthopedic surgical patients: Antithrombotic Therapy and Prevention of Thrombosis, 9th ed: American College of Chest Physicians Evidence-Based Clinical Practice Guidelines. Chest. 2012;141(2 Suppl):e227S-e277S.

23. Haut ER, Garcia LJ, Shihab HM, Brotman DJ, Stevens KA, Sharma R, Chelladurai Y, et al. The effectiveness of prophylactic inferior vena cava filters in trauma patients: a systematic review and meta-analysis. JAMA Surg. 2014;149(2):194-202.

24. Peterson EA, Yenson PR, Liu D, Lee AY. Predictors of attempted inferior vena cava filters retrieval in a tertiary care centre. Thromb Res. 2014;134(2):300-304. 